\title{
Orientación de la competencia digital del profesor universitario en las propuestas de integración de TIC
}

\author{
Orientation about digital competence on university professor throughout ICT integration proposals
}

\author{
Edgar Alfonso Pérez García \\ Rocío Adela Andrade Cázares
}

\begin{abstract}
\begin{tabular}{l|l} 
RESUMEN & ABSTRACT
\end{tabular}
Este trabajo muestra la orientación de la competencia digital del profesor universitario a partir de las propuestas de incorporación de TIC en su práctica docente, bajo el fundamento de la planeación estratégica. Se busca reconocer cómo es que el conocimiento, uso e intención que el docente considera tener en relación con las TIC reconfiguran sus propuestas de integración de tecnología. La metodología fue mixta concurrente por complementación, el diagnóstico fue cuantitativo mediante una encuesta en línea para obtener el nivel de intencionalidad, conocimiento, así como el uso de TIC, y se realizó un análisis documental de las propuestas de incorporación elaboradas por los profesores. Se obtiene que, aun cuando manifiestan tener un nivel medio de competencia digital y alto uso de recursos tecnológicos, estos factores no inciden en el desarrollo de las propuestas estratégicas de integración. Expresaron estar de acuerdo en la incorporación de tecnología a partir de la relación contenido-pedagogía-tecnología, sin embargo, se observa un desequilibrio, se aborda el componente tecnológico con alta prioridad y se identifican deficiencias en el pedagógico.

This paper shows the orientation of digital competence of university teachers through elaboration of proposals for incorporation of ICT in their teaching practice. It is based on the knowledge, use and intention that the teacher have regarding ICT, as phenomenon and influence in the process to elaborate his proposals for integration. The methodology was concurrent mixed by complementarity, we used diagnostic to obtain the level of intent, knowledge and use of ICT with an online questionnaire, and a documentary analysis was made of integration proposals creates by the teachers. Even when teachers have a medium level in digital competence and high use of ICT, this factors don't affect the design of strategic integration proposals. The teachers declare that they agree the incorporation of ICT from the content-pedagogytechnology components, however, an imbalance is observed, the technological component is addressed with high priority and deficiencies are identified in the pedagogical component.

Keywords: appropriation, digital competence, ICT, strategy, planning.
\end{abstract}

Palabras clave: apropiación, competencia digital, estrategia, planeación, TIC. 


\section{INTRODUCCIÓN}

La competencia digital de los docentes como objeto de estudio, el desarrollo como proceso y la incorporación de tecnologías de la información y comunicación (TIC) son temas que han cobrado relevancia dentro del entorno educativo (Durán, Gutiérrez y Prendes, 2016; Gisbert, González y Esteve, 2016). Acerca de estos temas se han documentado importantes trabajos que van desde la conceptualización, evolución histórica, propuesta de modelos y revisiones documentales (Sánchez y Navío, 2018). Respecto a la incorporación/integración/uso de TIC en la práctica pedagógica las consideraciones se orientan desde lo instrumental hasta planteamientos integradores entre la dimensión técnica, pedagógica y disciplinar (Forestello, 2013; Montero, 2010; Prendes, Gutiérrez y Martínez, 2018), generalmente empujados por iniciativas institucionales que exigen al profesor la incorporación.

El tema de "competencia digital docente se ha convertido en un elemento clave para la construcción del conocimiento pedagógico útil para la práctica y la mejora del aprendizaje de los estudiantes" (Benali, Kaddouri y Azzimani, 2018, p. 99), también para la formación de profesores (UNIR, 2018), y además son pocos los modelos de referencia que los guían sobre las áreas o dimensiones a desarrollar, los niveles y las perspectivas (pedagógica y/o instrumental) desde donde se hace la incorporación de las TIC (INTEF, 2017; Nolasco y Ramírez, 2011; Rangel, 2015), hecho que fomenta el desarrollo de la competencia digital (Prendes, Gutiérrez y Martínez, 2018).

Con base en este escenario, el propósito de este trabajo es reconocer cómo la relación del conocimiento/uso que el profesor considera tener de las TIC influye en la elaboración de propuestas de integración de tecnologías en su práctica docente a partir de un proceso de planeación estratégica y de selección de tecnología, y cómo esta incorporación aporta al desarrollo de la competencia digital docente en el contexto de una institución de educación superior pública en México.

Edgar Alfonso Pérez García. Director de Educación a Distancia y profesor hora clase de la Facultad de Ingeniería de la Universidad Autónoma de San Luis Potosí, México. Maestro en Ingeniería por la Facultad de Ingeniería de la UASLP. Es doctorando del programa de Innovación en Tecnología Educativa de la Universidad Autónoma de Querétaro. Correo electrónico: edgarperez@uaslp.mx. ID: https://orcid.org/0000-0003-4923-8266.

Rocío Adela Andrade Cázares. Docente-investigadora de la Facultad de Psicología de la Universidad Autónoma de Querétaro, México. Imparte las cátedras de Didáctica, Laboratorio de Docencia I y II, Evaluación del Aprendizaje, Evaluación de Procesos Psicoeducativos y otros cursos de posgrado. Es docente en la licenciatura en Innovación y Gestión Educativa, en el doctorado en Innovación en Tecnología Educativa (DITE-UAQ) y en el doctorado en Educación Multimodal (DEM-UAQ). Miembro del Consejo Mexicano de Investigación Educativa y de la Red Mexicana de Investigadores de la Investigación Educativa (REDmiIE). Correo electrónico: rocio.andrade@uaq.mx. ID: https://orcid.org/0000-0002-3966-9882. 


\section{Competencia digital docente}

En la actualidad es común encontrar dentro de los modelos educativos y planes de desarrollo (como referentes institucionales) las competencias docentes que dan identidad y claridad a las necesidades de formación de los profesores. En la definición de estas competencias es requerida la que está en relación con el uso de TIC, tecnologías o entornos digitales.

Esta tendencia ha desatado la delimitación de al menos tres aproximaciones, el uso, la incorporación y la apropiación de TIC, además de variadas estrategias de formación, con la finalidad de brindar a los profesores los insumos y elementos necesarios para el desarrollo correcto de su práctica docente a través de escenarios o medios digitales.

La definición del concepto de competencia digital docente todavía es difusa, sin embargo, las aproximaciones reflejan primero la concreción de competencia digital (Durán, Gutiérrez y Prendes, 2016) en general para cualquier individuo que hace uso de TIC o herramientas en medios digitales y posteriormente se incorpora el componente pedagógico-didáctico desde donde se relaciona o acota el ámbito de actuación del individuo.

En este sentido, la propuesta de Pérez y Andrade (2018) de los componentes que estructuran la competencia digital docente relaciona las competencias genéricas, las docentes y la digital; aun cuando la perspectiva es muy general, la propuesta da forma, comienza a delimitar y establecer los conocimientos o habilidades intrínsecas al concepto.

En la propuesta presentada por Durán, Gutiérrez y Prendes (2016) los componentes que definen las características de la competencia digital del profesor universitario específicamente son: 1) la competencia TIC general que integra las dimensiones de comunicación, información, multimedia, tecnológica, seguridad y resolución de problemas; 2) la competencia TIC del docente, que incorpora aspectos propios de la labor como gestión, formación, evaluación, potencial didáctico y facilitación del aprendizaje, y 3) dimensiones específicas como la publicación y difusión de material en red y la investigación e innovación pedagógica para el uso de TIC.

Es importante hablar de competencia digital docente a partir de sus componentes, así es posible reconocer las áreas o dimensiones para percibir el enfoque y alcance, por un lado, como sustento para aquellos intentos que buscan examinar el nivel de desarrollo, por otro, para dar claridad en la estructura conceptual, así como delimitar las dimensiones de los aspectos que intervienen (INTEF, 2017; Rangel, 2015; Valdivieso y Gonzáles, 2016); a partir de estos marcos referentes o modelos es posible diagnosticar, evaluar y encaminar los esfuerzos hacia una certificación (Durán, Prendes y Gutiérrez, 2019). 


\section{La integración de TIC}

Las TIC en el campo educativo han generado altas expectativas, tanto como apoyo a la presencialidad y como medio para fortalecer las alternativas de formación a las modalidades no presenciales. A partir de la incorporación de las TIC se han implementado acciones de formación y evaluación que permiten valorar o avalar el uso apropiado dentro de las distintas áreas educativas como la docente, curricular, administrativa, tutoral, etc.

Específicamente en el área docente se han planteado propuestas que van desde el uso, incorporación, integración y apropiación de TIC, denotando en cada uno de estos esquemas un nivel distinto de aplicación, de objetivo mediático de los materiales o herramientas y de la reconfiguración que la práctica pedagógica que se provoca por la misma incorporación de TIC generada desde una perspectiva reflexiva, planeada, crítica y evaluada.

En este sentido, la reflexión en el trabajo de Paredes (2012) se da en relación a la integración y no desde la incorporación de las TIC, que tiene como punto de partida la planificación estratégica donde se analizan cuestiones de infraestructura, oferta académica, capacitación y recursos digitales, es decir, las variables que aportan cada uno de estos elementos se analizan desde una perspectiva coherente entre la pedagogía y la tecnología, con el objetivo de dar certidumbre en su integración (Ángeles, 2012), siempre y cuando la planeación estratégica sea "entendida como un proceso donde se sistematiza y crean planes que proponen estrategias orientadoras, evaluables a través de indicadores, del quehacer dentro de la organización y con horizontes de tiempo concreto" (Paredes, 2012, p. 4). Las bases para la planeación estratégica usadas están fundamentadas en tres etapas: 1) la construcción de una visión estratégica, 2) la planificación y 3) la implementación (Paredes, 2012).

En este sentido, el planteamiento de Nóbile y Sanz (2014) es jerárquico, a partir de: 1) el país, donde se revisan las políticas públicas y las decisiones tomadas por el Estado sobre las TIC; 2) la institución, tomando en cuenta la cultura, la infraestructura tecnológica y física, la organización y las estrategias para la incorporación de tecnologías, y 3) el docente, a partir de su acceso, adopción, adaptación, apropiación e invención respecto al uso de las TIC en su práctica pedagógica, con este contexto es posible indagar el nivel de integración de TIC.

Otra perspectiva la menciona Ángeles (2012), quien considera la planeación como estrategia institucional a través de: 1) un diagnóstico inicial; 2) la delimitación de objetivos; 3) el diseño de estrategias, políticas y programas; 4) la definición de proyectos; 5) la definición de indicadores; 6) el seguimiento; y 7) evaluación. El contexto se analiza a partir de las políticas universitarias, los cambios organizacionales, la infraestructura y las particularidades de la innovación docente. 
Aun cuando las estrategias comentadas previamente se realizan desde una perspectiva estratégica, se abordan de manera superficial o nula las variables relacionadas con los principales actores de la práctica docente (estudiantes, profesores y la institución); en el caso de Montero (2010), la estrategia de incorporación (no de integración) de TIC se da desde la perspectiva de algunos profesores solamente.

Con base en esta perspectiva, la estrategia planteada en este trabajo se realiza desde el contexto más cercano al profesor en relación con el estudiante y el entorno institucional, y además se toma en consideración el enfoque del área disciplinar (el qué), pedagógico (el cómo) y técnico (a través de) con especial atención.

\section{La competencia digital docente desde la incorporación de TIC}

La correcta incorporación de herramientas tecnológicas en la práctica docente debe estar considerada como apoyo directo al enfoque, modelo, metodología y dinámicas usadas para desarrollar el proceso de enseñanza-aprendizaje, por ello los procesos formativos del profesor como profesional deben estar ligados a la integración de TIC, de aquí la importancia de analizar las competencias digitales como parte de las competencias profesionales docentes (Prendes, Gutiérrez y Martínez, 2018).

Las consideraciones elementales para el desarrollo de las competencias digitales parten de su concepción general como:

Valores, creencias, conocimientos, capacidades y actitudes para utilizar adecuadamente las tecnologías, incluyendo tanto los ordenadores como los diferentes programas e internet, que permiten y posibilitan la búsqueda, el acceso, la organización y la utilización de la información con el fin de construir conocimiento [Durán, Prendes y Gutiérrez, 2019, p. 190].

Este conjunto de características intrínsecas (conocimiento, habilidades, capacidades, creencias, actitudes) generan en los profesores la necesidad de conocer diversos dispositivos, medios o servicios digitales para comunicación, interacción, colaboración, búsqueda e intercambio de información, llevándolos a redefinirse como ciudadanos en entornos físicos y virtuales a través del desarrollo o adecuación de sus derechos, comportamientos, obligaciones, y de pensamiento (Pérez y Andrade, 2018).

A partir del contexto anterior se infiere que los programas de formación o las estrategias generales para la integración de TIC en la práctica docente son variados, en cada intento se toman diferentes aspectos, que en la mayor parte de las ocasiones no coinciden con el contexto particular del profesor, del estudiante, los contenidos o la institución. Por esta razón, la relación entre tecnología y educación debe realizarse desde la reflexión, crítica, planeación y evaluación, con el objetivo de orientar las propuestas de uso de TIC hacia una integración, es decir, crear una articulación recíproca en la cual ambas contribuyen al logro de un objetivo común; aquí es donde la planeación estratégica busca asegurar el éxito (Paredes, 2012). 
En este sentido, Prendes, Gutiérrez y Martínez (2018) mencionan que el mismo docente es quien debe decidir cuál es el rol de las tecnologías en su práctica, por ello necesita "diagnosticar situaciones de enseñanza, decidir el tratamiento y aplicarlo, lo que conlleva una formación y dedicación acorde con sus responsabilidades" (p. 5) con el fin de integrar las TIC de forma coherente en su función docente y así desarrollar su propia competencia digital.

\section{Propuesta de incorporación de TIC}

Las propuestas para la integración de tecnologías creadas por los profesores universitarios durante el desarrollo de este trabajo se basa en la propuesta de Paredes (2012), que combina la reflexión de la teoría con el saber pedagógico, el tecnológico, a partir del conjunto de aspectos académico-administrativos establecidos por el contexto; esta referencia teórica se basa en el TPACK Framework, en el cual se hace alusión al conjunto de conocimientos sobre las complejas relaciones entre la tecnología, la pedagogía y los contenidos específicos de cada escenario de actuación docente (Harris, Phillips, Koehler y Rosenberg, 2017).

$\mathrm{El}$ proceso de formulación de las propuestas realizadas por los profesores, basadas en la planeación estratégica, busca evitar o reducir al máximo la simulación, así como el uso improvisado de las TIC. Para ello se reconoce que las estrategias de formación deben trascender a la simple instrumentación de las tecnologías, recursos y servicios digitales. Deben tomar en cuenta la diversidad de intereses, problemáticas y expectativas como parte del contexto de actuación docente (Rodríguez, 2012).

Es por ello que la propuesta de formación para la integración de TIC considera dos aspectos esenciales, el primero inicia desde la planeación del curso (figura 1), concebida como la articulación entre lo que se pretende enseñar (el qué) y los mecanismos con los que se pretende lograr el aprendizaje (el cómo). Durante este proceso se toman decisiones particulares que definen las teorías, metodologías y/o enfoques a través de los cuales se busca obtener los mejores resultados de aprendizaje, es decir, a partir de la planeación didáctica se genera un proceso de enseñanza que garantice la cobertura curricular de acuerdo con el contexto donde se desarrolle (Amaro, 2011).

A partir de la relación (figura 1) entre el contenido y los mecanismos pedagógicos (desde la perspectiva de TPACK Framework) establecidos, el componente tecnológico fungirá por un lado como un mediador sobre todas aquellas acciones directas (interacción, intercambio de información, etc.), y por otro como un agente que reconfigurará las dinámicas de acción entre los mecanismos pedagógicos y los actores. El resultado logra mantener el mismo objetivo central sin que la tecnología tome relevancia sobre los otros dos componentes. 


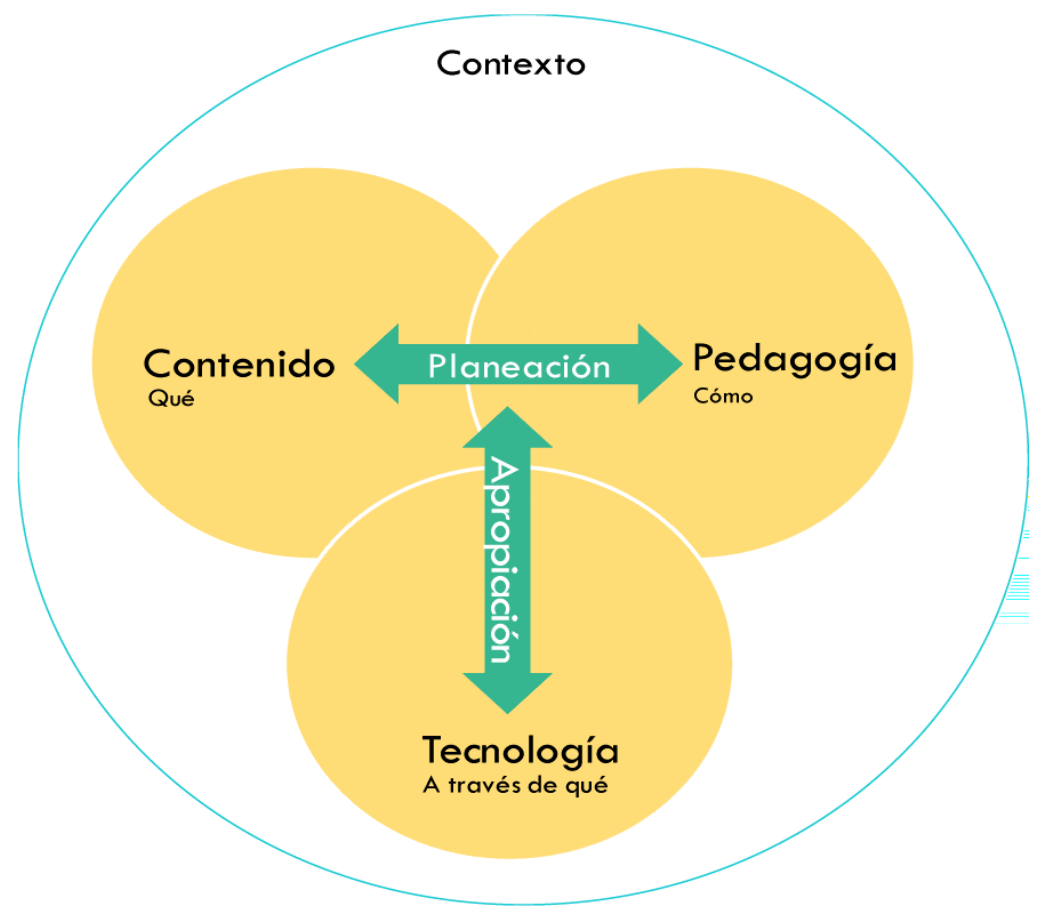

Figura 1. Relación entre los componentes del TPACK Framework, la planeación y la apropiación tecnológica.

Fuente: Elaboración propia a partir de Koehler, Mishra, Kereluik, Seob y Graham, 2014.

El otro aspecto tiene que ver con los factores inmersos durante el proceso de selección de tecnología (tabla 1), es decir, todos aquellos recursos educativos digitales (materiales, herramientas, dispositivos, servicios) que se utilizarán durante la conducción del proceso de enseñanza-aprendizaje con base en el contenido, el estudiante y la misma institución (Escamilla, 2005).

A reserva de los elementos mencionados por Escamilla (2005), la planeación estratégica de este trabajo también considera el contexto del profesor como otro componente (tabla 2) que contiene aspectos que influyen al momento de seleccionar la tecnología.

De manera conjunta, la figura 2 reúne los actores, componentes, factores, relaciones, tensiones y objetivo de la práctica pedagógica del profesor con integración de TIC que propone un cambio innovador único de acuerdo con el contexto tan particular que se estructura del conjunto de variables de cada elemento, sin perder de vista el objetivo central.

Es así como, las propuestas de integración de tecnología se realizarán a partir de una propuesta innovadora basada en la planeación estratégica, sin embargo, se desconoce si el factor uso y conocimiento de las TIC por parte del profesor tendrá influencia suficiente durante la elaboración de las propuestas. 
Tabla 1. Elementos por considerar durante el proceso de selección de la tecnología.

\begin{tabular}{|c|c|c|c|}
\hline Elemento & Aspecto & \multicolumn{2}{|c|}{ Orientación } \\
\hline$\overline{\text { Contenido }}$ & Estructura & \multicolumn{2}{|c|}{ La naturaleza de la materia de estudio } \\
\hline Se analiza el área del conocimiento & Presentación & \multicolumn{2}{|c|}{ Abstractas } \\
\hline y el tipo de curso & & \multicolumn{2}{|l|}{ Concretas } \\
\hline & Variación al interior & \multicolumn{2}{|l|}{ Global } \\
\hline & & \multicolumn{2}{|l|}{ Específica } \\
\hline & Planeación del proceso E-A & \multicolumn{2}{|l|}{ Objetivos } \\
\hline Estudiante & Edad & \multicolumn{2}{|l|}{ Madurez } \\
\hline Se realiza una reflexión sobre el & & \multicolumn{2}{|l|}{ Desarrollo intelectual } \\
\hline estudiante que toma el curso & & \multicolumn{2}{|l|}{ Nivel escolar } \\
\hline & Conocimientos previos & \multicolumn{2}{|c|}{ Diagnóstico de conocimientos previos } \\
\hline & & \multicolumn{2}{|l|}{ Uso de tecnología } \\
\hline & Estilos de aprendizaje & \multirow{4}{*}{ Ambiente } & Luz \\
\hline & & & Sonido \\
\hline & & & Temperatura \\
\hline & & & Asientos \\
\hline & & \multirow[t]{2}{*}{ Estímulos físicos } & Tiempo \\
\hline & & & $\overline{\text { Modalidad }}$ \\
\hline & & \multirow[t]{3}{*}{ Estructura y soporte } & Monitoreo \\
\hline & & & Motivación \\
\hline & & & Tipo de actividad \\
\hline & \multicolumn{3}{|l|}{ Nivel socio económico } \\
\hline & \multicolumn{3}{|l|}{ Cultura } \\
\hline & \multicolumn{3}{|l|}{ Acceso a la tecnología } \\
\hline Contexto institucional & \multicolumn{3}{|l|}{ Localización } \\
\hline Reflexión sobre las características & \multirow[t]{2}{*}{ Modalidad } & \multicolumn{2}{|l|}{ Escolarizada } \\
\hline del contexto institucional & & \multicolumn{2}{|l|}{ Abierta } \\
\hline & \multirow[t]{4}{*}{ Recursos institucionales } & \multicolumn{2}{|l|}{ Tecnología disponible } \\
\hline & & \multicolumn{2}{|l|}{ Recursos económicos } \\
\hline & & \multicolumn{2}{|l|}{ Recursos humanos } \\
\hline & & \multicolumn{2}{|c|}{ Postura sobre uso de tecnología } \\
\hline
\end{tabular}

Fuente: Elaboración propia a partir de Escamilla (2005).

Tabla 2. Aspectos relacionados con el contexto del profesor.

\begin{tabular}{lc}
\hline \multicolumn{1}{c}{ Elemento } & Aspecto \\
\hline Profesor & Disponibilidad de TIC \\
Contexto del profesor para la incorporación de TIC & Práctica docente actual \\
& Materiales digitales disponibles \\
& Infraestructura disponible \\
\hline
\end{tabular}




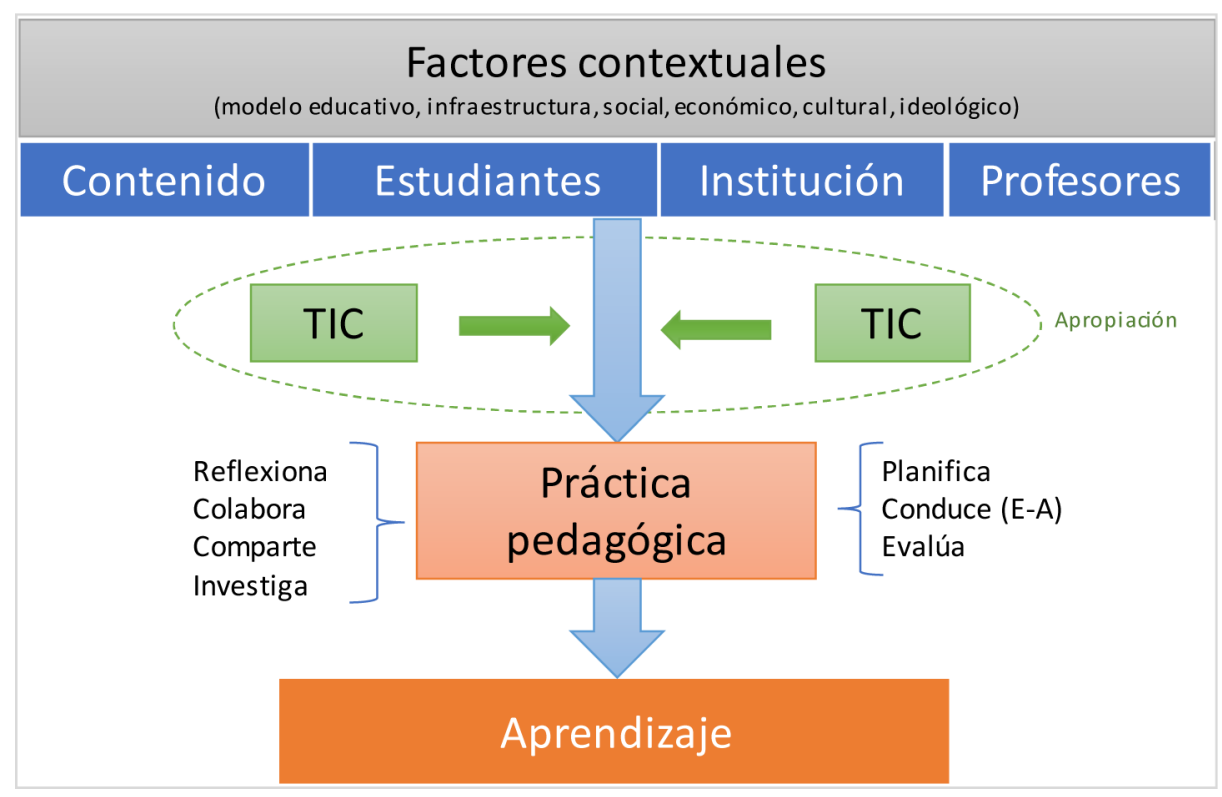

Figura 2. Relación entre actores, factores y la apropiación de TIC en la práctica pedagógica.

Fuente: Elaboración propia.

\section{Estrategia para la creación de propuestas para la incorporación de TIC}

Con la intención de lograr el objetivo planteado en este trabajo se propuso un proceso en el cual intervienen tres etapas:

- Diagnóstico. Con el objetivo de recabar información contextual del profesor y su percepción acerca de: 1) el nivel de utilidad de las TIC en la práctica docente en general; 2) la apropiación que estima tener sobre los recursos educativos digitales y las herramientas de comunicación, interacción e intercambio de información, y 3) la intencionalidad en el uso de materiales y herramientas digitales en sus propuestas de integración de TIC.

- Formación. Una vez reunidos los profesores, se les contextualizó, se informó y se hizo explícito el objetivo de la estrategia. Esta etapa se desarrolló en dos partes, la primera se conformó con la revisión y conceptualización de cada uno de los elementos considerados en la planeación estratégica, selección de tecnología, incorporación y apropiación de TIC; la segunda etapa consistió en la revisión técnica de materiales digitales y herramientas enfocadas a la comunicación, colaboración, intercambio de información, interacción, con el objetivo de analizar su alcance, ventajas, desventajas, limitaciones, así como las opciones para su integración en su práctica docente.

- Elaboración de propuestas y presentación en plenaria. En la primera parte de esta etapa el profesor reflexionó y analizó el contexto de los componentes propuestos para la selección de la tecnología y dio forma a su propuesta de integración de TIC. En una segunda parte, el profesor presentó en plenaria 
su propuesta ante el resto de los profesores participantes, quienes dieron retroalimentación y emitieron comentarios a las propuestas.

\section{Metodología}

El desarrollo metodológico seguido en este trabajo fue mixto, como un tercer paradigma que "permite combinar técnicas, métodos, conceptos o terminología en un solo estudio" (Burke y Onwuegbuzie, 2004, p. 17), con un diseño concurrente debido a que los datos recolectados en la fase cualitativa y cuantitativa se reúnen de manera simultánea para brindar una sola interpretación (Burke y Onwuegbuzie, 2004; Pole, 2009), la relación establecida fue QUAN + QUAL, a través de la estrategia de complementación con el propósito de recuperar distintas imágenes del escenario de actuación (Bericat, 1998). Con base en esto, la primera parte fue cuantitativa a través del uso de la técnica de encuesta como instrumento de recolección de datos. La validación del instrumento se realizó en dos partes, la inicial fue por expertos basada en la propuesta de Escobar y Cuervo (2008) con énfasis en la suficiencia, claridad, coherencia y relevancia, el área de desarrollo de los expertos fue la incorporación de tecnología en la enseñanza; la segunda parte consistió en la aplicación en una prueba piloto con la finalidad de ajustar y eliminar aspectos intrascendentes (Gómez, 2012). La aplicación del instrumento se efectuó en línea a través del servicio de Microsoft Forms, disponible como parte del conjunto de aplicaciones vinculadas al correo electrónico que asegura disponibilidad sin pérdida de datos. La escala utilizada en el instrumento para especificar el nivel de utilidad, uso e intencionalidad respecto a los materiales, herramientas y servicios digitales fue: 0 - nulo, 1 - bajo, 2 - medio, 3 - bueno y 4 - destacado.

La segunda parte del trabajo fue cualitativo, se realizaron procesos de interpretación y deducción a partir de las técnicas de revisión documental para la comprensión de significados (Canales, 2006; Hernández, Fernández y Baptista, 2006) en los documentos y materiales (presentaciones y sitios web principalmente) desarrollados por profesores, en los que estructuraron sus propuestas de integración de TIC en su práctica docente. El objetivo de esta etapa fue identificar el conjunto de aspectos y componentes involucrados en la práctica pedagógica que fueron tomados en cuenta para la elaboración de las propuestas.

El estudio se realizó a partir de una convocatoria abierta al interior de la institución para todos los profesores dispuestos a mejorar su práctica docente a través de la incorporación de TIC. Se reunieron 28 profesores con las características descritas en el tabla 3; de estos, 16 son mujeres y 12 hombres, 8 profesores son hora clase y 20 de tiempo completo. Las áreas del conocimiento a las que pertenecen los profesores se muestran en el tabla 4. 
Con el propósito de resguardar el anonimato de los profesores, se clasificaron por número de participante a dos dígitos, el tipo de nombramiento (PTC para profesor de tiempo completo y PHC para profesor hora-clase) y un guión medio seguido por el género (M para masculino, F para femenino), por ejemplo, "28PTC-M" refiere al participante número 28 , profesor de tiempo completo, masculino.

Tabla 3. Características generales de la muestra de profesores participantes.

\begin{tabular}{lccc}
\hline & $\begin{array}{c}\text { Edad } \\
\text { (en años) }\end{array}$ & $\begin{array}{c}\text { Cursos tomados relacionados con TIC } \\
\text { (últimos tres años) }\end{array}$ & $\begin{array}{c}\text { Cursos tomados relacionados con aspectos } \\
\text { pedagógicos (últimos tres años) }\end{array}$ \\
\hline Media & 41.64 & 2.71 & 1.86 \\
\hline Deviación estándar & 6.53 & 1.80 & 1.69 \\
\hline Rango & $30-59$ & $1-8$ & $0-6$ \\
\hline
\end{tabular}

Fuente: Elaboración propia.

Tabla 4. Distribución de profesores por área de conocimiento.

\begin{tabular}{cc}
\hline Área & No. de profesores \\
\hline Biología y Química & 6 \\
\hline Biotecnología y Agropecuarias & 6 \\
\hline Ciencias de la Ingeniería & 5 \\
\hline Física, Matemáticas y Ciencias de la Tierra & 2 \\
\hline Humanidades y de la Conducta & 1 \\
\hline Investigación Multidisciplinaria & 1 \\
\hline Medina y Salud & 2 \\
\hline Sociales y Económicas & 5 \\
\hline
\end{tabular}

Fuente: Elaboración propia.

\section{ANÁLISIS Y RESULTADOS}

Se destaca la importancia que dan las y los profesores a los temas relacionados con TIC, ya que tienen un antecedente de formación mayor en temas de tecnologías que en aspectos relacionados con el componente pedagógico. Los resultados muestran que aun cuando el nivel de disponibilidad de incorporación de TIC de las y los docentes es destacado, en la práctica el uso en general, personal, con sus estudiantes y colegas, disminuye (figura 3).

En relación con las distintas áreas competenciales los profesores consideran en general tener el mismo nivel de desarrollo (tabla 5). En este sentido se considera destacada la capacidad de creación de materiales digitales, excepto para cursos masivos abiertos y en línea (MOOC por sus siglas en inglés), sin embargo la importancia y el uso de estos materiales digitales en las propuestas de integración de TIC dismi- 


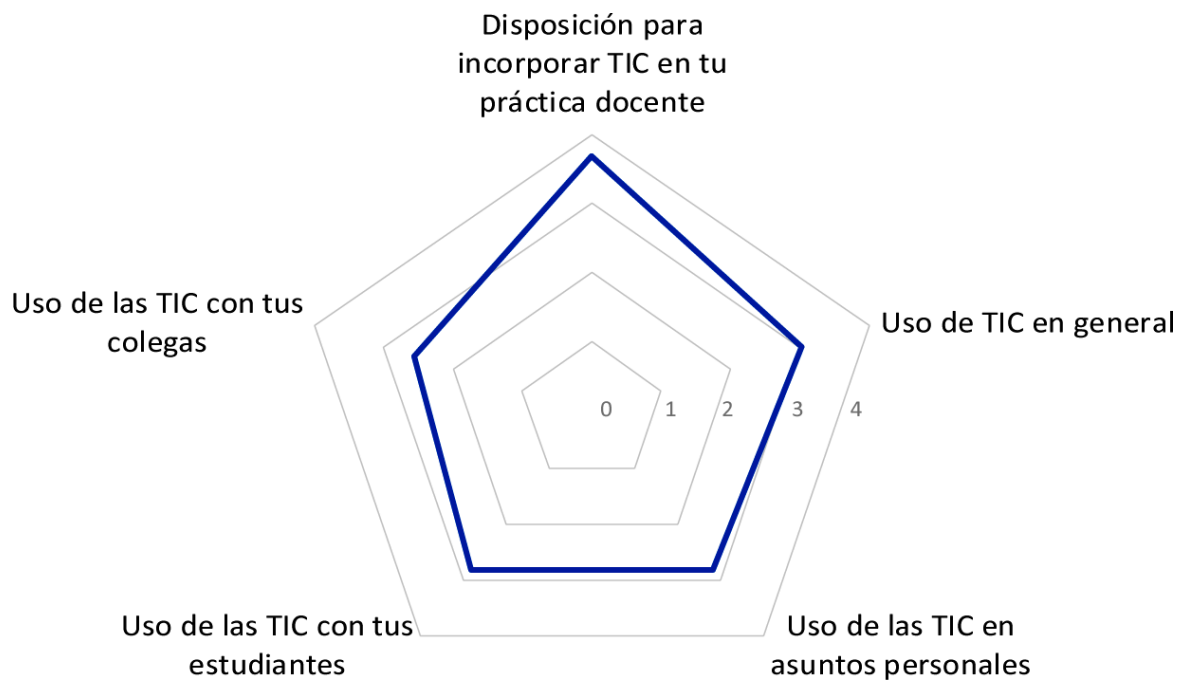

Figura 3. Percepción de disponibilidad y uso de TIC en los profesores.

Fuente: Elaboración propia.

Tabla 5. Perspectiva del profesor para el uso de TIC en sus propuestas.

\begin{tabular}{|c|c|c|c|c|c|c|}
\hline \multirow{4}{*}{$\frac{\text { Aspecto }}{\text { Uso general en la propuesta }}$} & \multicolumn{6}{|c|}{ Material o herramienta } \\
\hline & \multirow[b]{2}{*}{ Materiales } & \multicolumn{3}{|c|}{ Intercambio } & \multirow{2}{*}{\multicolumn{2}{|c|}{$\begin{array}{c}\text { Búsqueda } \\
\text { de información }\end{array}$}} \\
\hline & & Comunicación & de información & Colaboración & & \\
\hline & 3 & 3 & 3 & 3 & & 3 \\
\hline \multirow[t]{2}{*}{ Importancia y uso de TIC } & Imágenes & Audios & Videos & Presentaciones & $\mathrm{OA}$ & MOOC \\
\hline & 4 & 3 & 4 & 4 & 3 & 3 \\
\hline \multirow{2}{*}{$\begin{array}{l}\text { Capacidad para desarrollo } \\
\text { de materiales }\end{array}$} & Imágenes & Audios & Videos & Presentaciones & $\mathrm{OA}$ & MOOC \\
\hline & 4 & 4 & 4 & 4 & 4 & 3 \\
\hline \multirow[t]{2}{*}{ Uso de comunicación asíncrona } & Foros & Redes sociales & Correo electrónico & \multicolumn{3}{|c|}{ Otros } \\
\hline & 3 & 3 & 3 & \multicolumn{3}{|c|}{3} \\
\hline \multirow[t]{2}{*}{ Uso de comunicación instantánea } & Facebook & WhatsApp & Skype & Hangouts & \multicolumn{2}{|c|}{ Otros } \\
\hline & 2 & 3 & 2 & 2 & \multicolumn{2}{|c|}{3} \\
\hline \multirow[t]{2}{*}{ Intercambio de documentación } & Nube & Correo electrónic & Espacios virtuales & \multicolumn{3}{|c|}{ Otros } \\
\hline & 3 & 3 & 4 & \multicolumn{3}{|c|}{3} \\
\hline
\end{tabular}

Fuente: Elaboración propia.

nuye. Es sobresaliente la intención de uso para imágenes, presentaciones y videos principalmente, dejando los audios, objetos de aprendizaje y MOOC en un segundo término. Existe una marcada diferencia de uso entre los mecanismos de comunicación asíncrona y síncrona independiente del medio que se disponga a utilizar, es decir, no influye el medio sino la dinámica de comunicación que se requiere.

En el ámbito de materiales digitales y mecanismos de comunicación, en la figura 4 se muestra: a) que las principales ventajas del uso de recursos educativos digitales 
son la rapidez, la mejora, se centran en el aprendizaje, la facilidad, la disponibilidad. En referencia a la codificación de los participantes, mencionada en la metodología, uno de los profesores cita que "mejora participación, mejor comprensión de temas, clases más amenas” (28PTC-M). Por el contrario, en b) se reúnen los términos que tienen representatividad en la problemática para el uso de recursos; sobresalen: internet, acceso, limitación, elaborar, plataforma. El comentario de uno de los participantes fue: "Peso de los recursos. Acceso a internet fuera de la UASLP [Universidad Autónoma de San Luis Potosí] por parte de algunos alumnos. Falta de tiempo para la planeación, diseño y desarrollo de materiales" (20PTC-M).

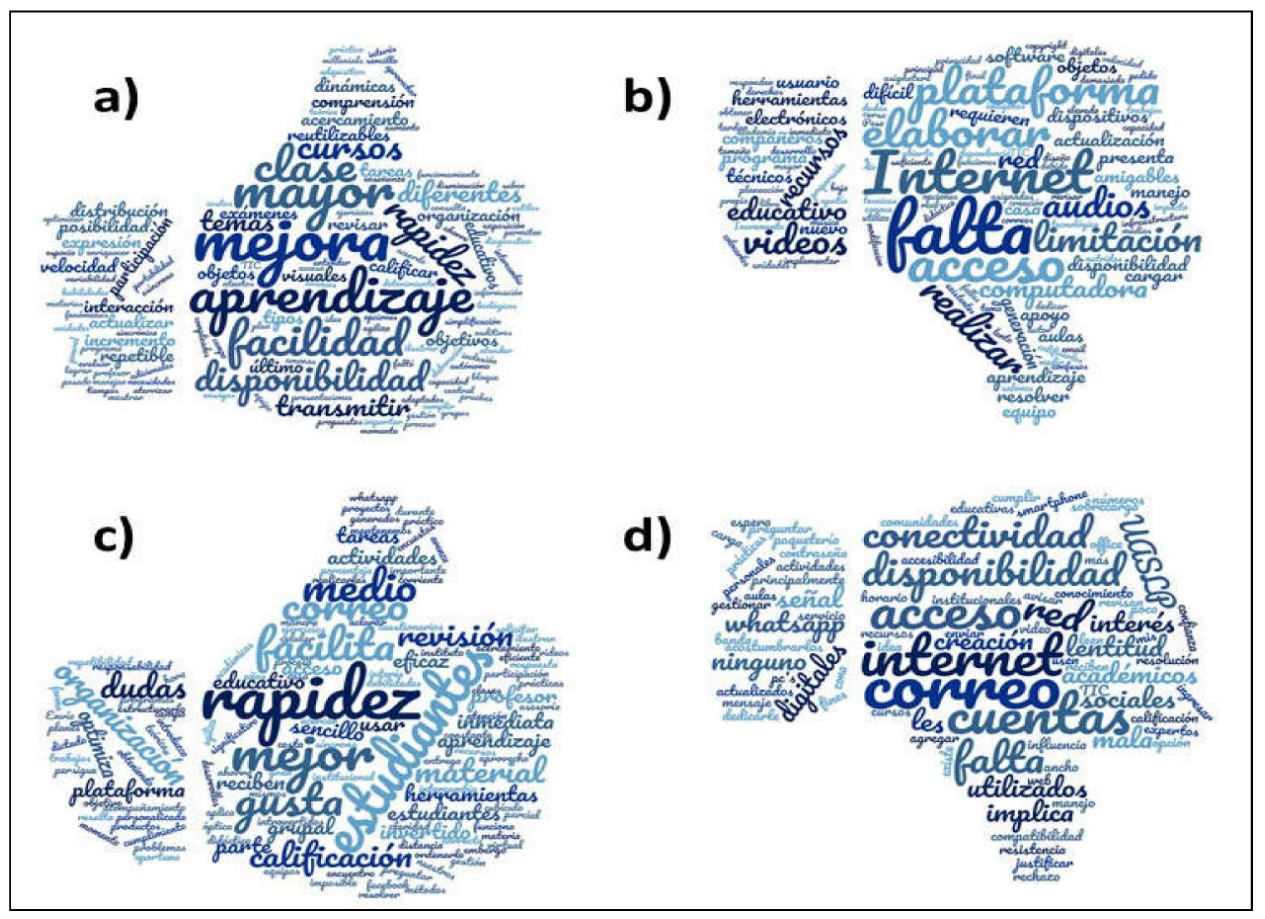

Figura 4. Problemas y las ventajas en el uso de materiales educativos y comunicación.

Fuente: Elaboración propia.

Respecto al tema de comunicación, c) destaca las ventajas, entre las que sobresale la rapidez, facilidad, mejora, revisión y los estudiantes; la cita textual de uno de los participantes dice: "La revisión de los productos generados es más sencilla, la comunicación se puede dar en cualquier momento" (13PTC-F). Por último, d) muestra que las principales desventajas son las dificultades de acceso a internet, correo, cuentas, disponibilidad, conectividad; una referencia textual fue: "El acceso a internet de mis estudiantes, pero en general no hay problema con la plataforma y el correo electrónico" (05PTC-F).

A partir de la relación entre la estimación que el profesor considera en el uso y apropiación de tecnologías y sus propuestas de integración se obtiene que los recursos 
más utilizados son los videos (94\%), imágenes y presentaciones (78\%), seguidas por objetos de aprendizaje (56\%), cabe destacar que el uso de los MOOC no se prevé en ninguna de las propuestas.

Con respecto a las herramientas de comunicación e intercambio de información, los espacios virtuales se destacan de manera sobresaliente con el $94 \%$ de uso, otros mecanismos como el correo electrónico, redes sociales, Facebook, como máximo tienen una consideración de no más del 22\%, y los mecanismos de comunicación síncrona como Whats App, Skype y Hangouts no fueron considerados por ningún profesor.

En relación con la selección de tecnología para elaborar sus propuestas, los resultados muestran que, en el componente de contenido, la estructura del programa y los objetivos del curso (ambos al 100\%) son los aspectos más relevantes, seguidos por la incorporación de tecnología en actividades específicas. Del componente de estudiantes, el acceso a la tecnología es el aspecto más relevante (72\%), el resto se ubica por debajo del 28\%, cabe destacar en este punto que el aspecto relacionado con el ambiente físico no fue considerado en ninguna de las propuestas. Respecto al contexto institucional la tecnología disponible es el aspecto más relevante, tomado en cuenta en un 67\%, y el resto por debajo del 39\%. Por último, en los aspectos relacionados con los profesores, la disponibilidad de TIC (50\%) y sus materiales digitales $(39 \%)$ fueron los que más se consideraron.

\section{Discusión}

Los aspectos más relevantes que surgen a partir de la información brindada por el profesor y complementada con la revisión de las propuestas tiene que ver con la planeación de la práctica docente, si bien los profesores estiman tener en promedio entre uno y dos cursos formativos sobre aspectos pedagógicos, la observación del grupo y los documentos entregados indican deficiencias al desarrollo de su propia planeación. Por otra parte, los profesores prefieren la formación en temas relacionados con TIC que en temas pedagógicos (planeación, evaluación, etc.), de acuerdo con los datos de la encuesta.

Aun cuando el profesor está consciente de la incorporación de la tecnología con base en los términos del TPACK (disciplina-pedagogía-tecnología), los resultados muestran que en general deben resolverse problemas del área pedagógica (formación, instrucción o actualización); se infiere que el componente mejor desarrollado es el disciplinar, seguido por el técnico y finalmente el pedagógico. Este escenario muestra un desequilibrio entre las tensiones de cada componente y por ende la incorporación de tecnología dentro del ambiente de aprendizaje impide lograr la correcta integración de TIC en la práctica docente.

En este sentido, los profesores dan prioridad al uso de la tecnología en su práctica docente antes de preguntarse cómo lograr los objetivos de aprendizaje, esta práctica 
se contrasta con lo planteado en este trabajo, el cual considera que la adopción estratégica de la tecnología parte de la relación entre el componente disciplinar (el qué) y el pedagógico (el cómo).

En la selección de tecnología se observa que todos los aspectos relacionados con el componente de contenido y la institución fueron tomados en cuenta en mayor o menor grado durante la elaboración de las propuestas de integración de TIC, sin embargo, del componente de estudiantes, específicamente los que tienen que ver con el ambiente físico no fueron tomados en cuenta, sin embargo el alcance de este estudio no permite conocer las razones de ello.

Además de los tres componentes planteados por Escamilla (2005), este trabajo propuso incluir aspectos relacionados con el contexto del profesor; los resultados muestran que cada uno de estos se tomó en cuenta, por lo que se infiere que el contexto más cercano al profesor define también el comportamiento de la incorporación de tecnología.

De acuerdo con los resultados, las áreas competenciales de comunicación, interacción e intercambio de información muestran una disparidad en relación con el nivel de competencia digital que el profesor manifiesta tener. Un escenario similar ocurre con los materiales: los profesores consideran tener un potencial sobresaliente para el uso y la creación, sin embargo el uso dentro de las propuestas es de bajo a nulo, principalmente para los audios y los MOOC. Se destacan los videos como el recurso educativo digital con mayor uso dentro de las propuestas, seguido por presentaciones e imágenes.

En el área competencial de comunicación, el uso de TIC para una comunicación asíncrona refleja un mejor escenario de uso por parte de los profesores, se infiere que la familiaridad con el correo electrónico tiene un peso considerable; respecto al conjunto de herramientas para comunicación síncrona, Whats App es la herramienta que prevén usar.

\section{Conclusiones}

Los resultados y la discusión permiten concluir que, aun cuando las y los docentes tienen una destacada intención de usar tecnologías y manifiestan tener un buen manejo de estas, la planeación crítica, reflexiva, contextualizada y argumentada hace que sean selectivos con las tecnologías y se visualiza un uso racional y moderado tanto en materiales como en herramientas, esto disminuye la simulación en la práctica docente. Este proceso favorece el desarrollo de la competencia digital docente.

A partir de la planeación estratégica, la incorporación y selección de TIC para la mejora de la práctica docente no se desarrolla al mismo nivel entre los profesores, no tiene los mismos objetivos, enfoques o dinámicas, es decir, en cada docente la propuesta de integración es diferente, sin embargo su continuidad provoca cambios 
de mejora graduales e incrementables, por ello se da al profesor la flexibilidad de establecer su propio ritmo de integración de TIC.

Los aspectos relacionados al contexto del profesor deben ser considerados como parte de las variables de importancia durante la selección e incorporación de TIC en la práctica docente, es decir, su disponibilidad, la manera en que se imparten las clases, sus materiales y la infraestructura de la que disponen influyen en la definición de cuáles herramientas usarán y el grado de uso.

Es posible concluir que el conocimiento o uso que el profesor estima tener respecto a las TIC no es un factor que influye en los escenarios de incorporación de tecnologías en la práctica docente, se infiere que existe otro conjunto de factores que no favorecen u obstaculizan la puesta en práctica de dinámicas de comunicación, intercambio de información e incorporación de materiales digitales.

Aun cuando las y los docentes manifiestan que en las áreas competenciales de comunicación, materiales educativos y el intercambio de información tienen un uso o conocimiento intermedio, es posible concluir que la orientación se encamina hacia el producto (entrega o envío de documentos) y no hacia el proceso de aprendizaje.

\section{REFERENCIAS}

Amaro, R. (2011). La planificación didáctica y el diseño instruccional en ambientes virtuales. Investigación y Posgrado, 26(2), 129-160. Recuperado de: https:// bit.ly/2VyjZtT.

Ángeles, A. (2012). Planes estratégicos integrales para la incorporación y uso de TIC: claves para administrar el cambio. Razón y Palabra, (79). Recuperado de: https://www.redalyc.org/pdf/1995/199524411030. pdf.

Benali, M., Kaddouri, M., y Azzimani, T. (2018). Digital competence of Moroccan teachers of English. International Journal of Education and Development using Information and Communication Technology (IJEDICT), 14(2), 99-120.

Bericat, E. (1998). Estrategias y usos de la triangulación. En E. Bericat, La integración de los métodos cuantitativo y cualitativo en la investigación social: significado y medida. Barcelona: Ariel.

Burke, R., y Onwuegbuzie, A. (2004). Mixed methods research: A research paradigm whose time has come. Educational Researcher, 33(14), 14-26.

Canales, M. (2006). Metodología de la investigación social: introducción a los oficios. Santiago de Chile: LOM Ediciones.
Durán, M., Gutiérrez, I., y Prendes, M. P. (2016). Análisis conceptual de modelos de competencia digital del profesorado universitario. Revista Latinoamericana de Tecnología Educativa, 15(1), 97-114.

Duran, M., Prendes, M. P., y Gutiérrez, I. (2019). Certificación de la competencia digital docente: propuesta para el profesorado universitario. RIED, Revista Iberoamericana de Educación a Distancia, 22(1), 187-205.

Escamilla, J. G. (2005) Selección y uso de la tecnología educativa. México: Trillas.

Escobar, J., y Cuervo, Á. (2008). Validez de contenido y juicio de expertos: una aproximación a su utilización. Avances en Medición, (6), 27-36.

Forestello, R. (2013). Algunas pistas para pensar la integración de las TIC en la enseñanza. Revista de Educación en Biología, 16(1), 7-14.

Gisbert, M., González, J., y Esteve, F. (2016). Competencia digital y competencia digital docente: una panorámica sobre el estado de la cuestión. Revista Interuniversitaria de Investigación en Tecnología Educativa, (0), 74-88.

Gómez, S. (2012). Metodología de la investigación. México: Red Tercer Milenio. 
Harris, J., Phillips, M., Koehler, M., y Rosenberg, J. (2017). TPCK/TPACK research and development: past, present and future directions. Australian Journal of Educational Techonology, 33(3), I-VIII.

Hernández, R., Fernández, C., y Baptista, P. (2006). Metodología de la investigación. México: McGraw-Hill.

INTEF [Instituto Nacional de Tecnologías Educativas y de Formación del Profesorado] (2017). Marco común de competencia digital docente. España: INTEF. Recuperado de: https://bit.ly/2xU2A5w.

Koehler, M. J., Mishra, P., Kereluik, K., Shin, T. S., y Graham, C. R. (2014). The technological pedagogical content knowledge framework. En Handbook of research on educational communications and technology (pp. 101-111). Nueva York: Springer.

Montero, J. L. (2010). Estrategia para la introducción de las tecnologías de la información y las comunicaciones. Revista Latinoamericana de Tecnología Educativa RELATEC, 9(1), 75-87.

Nóbile, C., y Sanz, C. (2014). Marco de análisis del nivel de integración de TIC en instituciones de educación superior. Trabajo presentado en el XX Congreso Argentino de Ciencias de la Computación, Buenos Aires, Argentina.

Nolasco, P., y Ramírez, A. (2011). Una aproximación a un modelo de certificación de competencias digitales docentes. Trabajo presentado en el XI Congreso Nacional de Investigación Educativa, UNAM, México.

Paredes, H. (2012). TIC en la educación: planeación estratégica. Trabajo presentado en el V Congreso Internacional y VI Encuentro de Estudiantes y Egresados de Docencia Universitaria "Universidad: Docencia, Investigación e Innovación”, Universidad de Nariño, Colombia.
Pérez, E. A., y Andrade, R. A. (2018). El desarrollo de las competencias digitales en la innovación educativa mediadas por TIC. Trabajo presentado en el XXVI Encuentro Internacional de Educación a Distancia, Guadalajara, Jalisco, México.

Pole, K. (2009). Diseño de metodologías mixtas. Una revisión de las estrategias para combinar metodologías cuantitativas y cualitativas. Renglones, Revista arbitrada en Ciencias Sociales y Humanidades, (60), 37-42. Recuperado de https://bit.ly/2zoawwt.

Prendes, M. P., Gutiérrez, I., y Martínez, F. (2018). Competencia digital: una necesidad del profesorado universitario en el siglo XXI. Revista de Educación a Distancia (RED), (56), 1-22.

Rangel, A. (2015). Competencias docentes digitales: propuesta de un perfil. Revista de Medios y Educación, (46), 235-248.

Rodríguez, J. (2012) Análisis sobre la integración en el sistema educativo de las TIC: proyectos institucionales y formación permanente. Teoría de la Educación. Educacióny Cultura en la Sociedad de la Información, 13(3), 129-144.

Sánchez, M. M., y Navío, A. (2018). Las competencias TIC de los profesores en la Universidad de la Sabana: la mirada de sus estudiantes. Revista Interuniversitaria de Investigación en Tecnología Educativa - RiiTE, (5), 84-101.

UNIR [Universidad Internacional de La Rioja] (2018). Competencias digitales docentes. Informe grupal. Recuperado de: http://www.unir.net.

Valdivieso, T. S., y Gonzáles, M. A. (2016). Competencia digital docente: ¿Dónde estamos? Perfil del docente de educación primaria y secundaria. El caso de Ecuador. Pixel-Bit: Revista de Medios y Educación, (49), 57-73.

Cómo citar este artículo:

Pérez García, E. A., y Andrade Cázares, R. A. (2020). Orientación de la competencia digital del profesor universitario en las propuestas de integración de TIC. IE Revista de Investigación Educativa de la REDIECH, 11, e905. doi: https:/ / doi.org/10.33010/ie_rie_rediech.v11i0.905. 